\title{
Wide-Range Correlated Color Temperature Light Generation From Resonant Cavity Hybrid Quantum Dot Light-Emitting Diodes
}

\author{
Kuo-Ju Chen, Chien-Chung Lin, Senior Member, IEEE, Hau-Vei Han, Chia-Yu Lee, Shih-Hsuan Chien, \\ Kuan-Yu Wang, Sheng-Huan Chiu, Zong-Yi Tu, Jie-Ru Li, Teng-Ming Chen, Xiuling Li, \\ Min-Hsiung Shih, Member, IEEE, and Hao-Chung Kuo, Fellow, IEEE
}

\begin{abstract}
This study presents extremely uniform colloidal quantum dot white light-emitting diodes (QD-WLEDs) that demonstrate a high color rendering index (CRI) and correlated color temperatures (CCTs) ranging from 2500 to $4500 \mathrm{~K}$. Experimental results indicate that the structure of the distributed Bragg reflector (DBR) containing a stopband in the UV region enhances the intensity output of both monochromatic QD-LEDs and QD-WLEDs by reflecting the unconverted UV light back onto the package to excite the QDs further. Furthermore, the angular CCT uniformity of the QD-WLEDs also improved considerably because of the dependence of the DBR structure on the incident angle. The angular CCT deviation in the range of $-70^{\circ}$ to $70^{\circ}$ decreased to $39 \mathrm{~K}$ and the CRI of the WLED is higher than 90. The high-CRI and uniform angular CCT QD-WLED containing the DBR demonstrates potential applicability as the lighting source of next-generation display devices and solid-state lighting.
\end{abstract}

Index Terms-GaN, light-emitting diodes (LEDs), quantum dots (QDs).

\section{INTRODUCTION}

$\mathbf{L}$ IGHT-emitting diodes (LEDs) have been developed extensively in the past decade because of their high efficiency, long lifetime, and stability in comparison to traditional incandescent and fluorescent lamps [1]-[4]. Currently, the most common technique for producing white LEDs (WLEDs) involves combining blue LEDs with yttrium aluminum garnet (YAG:Ce) phosphor because of high luminous efficiency and

Manuscript received September 17, 2014; revised January 21, 2015; accepted February 11,2015. This work was supported by the National Science Council in Taiwan under Grants NSC101-2221-E-009-046-MY3, NSC 103-3113-E-009001-CC2, and NSC 103-2917-I-009-179.

K.-J. Chen is with the Department of Photonics and Institute of ElectroOptical Engineering, National Chiao Tung University, Hsinchu 30010, Taiwan and also with the Department of Electrical and Computer Engineering, University of Illinois at Urbana-Champaign, Urbana, IL 61801 USA.

C.-C. Lin and K.-Y. Wang are with the Institute of Photonic System, National Chiao Tung University, Tainan 711, Taiwan (e-mail: chienchunglin@faculty.nctu.edu.tw).

H.-V. Han, C.-Y. Lee, S.-H. Chien, S.-H. Chiu, Z.-Y. Tu, J.-R. Li, and H.C. Kuo are with the Department of Photonics and Institute of Electro-Optical Engineering, National Chiao Tung University, Hsinchu 30010, Taiwan.

T.-M. Chen is with the Phosphors Research Laboratory, Department of Applied Chemistry, National Chiao Tung University, Hsinchu 30010, Taiwan.

$\mathrm{X}$. Li is with the Department of Electrical and Computer Engineering, University of Illinois at Urbana-Champaign, Urbana, IL 61801 USA.

M.-H. Shih is with the Department of Photonics and Institute of ElectroOptical Engineering, National Chiao Tung University, Hsinchu 30010, Taiwan, and also with the Research Center for Applied Sciences, Academia Sinica, Taipei 115, Taiwan.

Color versions of one or more of the figures in this paper are available online at http://ieeexplore.ieee.org.

Digital Object Identifier 10.1109/JSTQE.2015.2404877 low cost [5], [6]. These types of WLEDs have been commercialized and YAG:Ce has already been developed as a downconverting material; however, the problem of the instability of the color quality remains a critical challenge for phosphor based WLEDs [7]. In general, the characteristics of WLEDs such as the homogenous correlated color temperature (CCT) and high color rendering index (CRI) are considered the key factors that must be used in solid-state lighting (SSL). Therefore, several studies have proposed several structures, such as the patterned remote phosphor structure and $\mathrm{ZrO}_{2}$-type phosphor structure, for reducing the angular CCT deviation in WLEDs [8], [9]. Furthermore, to improve the color rendering ability of WLEDs, enhancing the CRI to more than 80 is an essential target that can be achieved by compensating the missing red component in phosphor-converted WLEDs. Consequently, some previous studies have used methods that involve adding red-light emitting phosphors or combining red LEDs to form a hybrid warm WLED package to satisfy strict lighting standards for indoor lighting [10]-[12]. However, the red-light emitting phosphors exhibit some severe limitations such as high cost, large stokes shift losses, and reabsorption phenomenon, resulting in reduced efficiency in WLEDs [13], [14].

Recently, colloidal quantum dots (QDs) have attracted considerable scientific attention because of their unique properties such as high quantum yield, minimal backscattering, size dependent tunable bandgap, and narrow emission linewidth [15]-[18]. Core-shell structures have been employed to enhance the quantum yield of QDs and derive high photoluminescent efficiency [19], [20]. Major QD applications are currently focused on thin film display, monochromatic displays, and WLEDs [21]-[25]. Regarding the display technology, methods such as transfer printing [21], mist coating [22], inkjet printing [23], and pulsespray coating [26] have been used for deriving uniform and independent RGB pixels. Furthermore, regarding WLEDs, previous studies have indicated that phosphor converted WLEDs mixed with red QDs could yield high color-rendering properties [27], [28]. Moreover, Nizamoglu et al. [29] developed warm WLEDs that incorporate green and red $\mathrm{CdSe} / \mathrm{ZnS}$ coreshell nanocrystals hybridized with InGaN-GaN blue chips. The balance between color quality and intensity of QD-LEDs are imperative concerns that must be addressed.

The present study designed multicolor LEDs by combining colloidal QDs and polymethylmethaccrylate (PMMA). The PMMA is a host material that is used to embed the QDs and provide a certain degree of isolation and protection. Furthermore, $\mathrm{HfO}_{2} / \mathrm{SiO}_{2}$ distributed Bragg reflector (DBR) with a stopband 


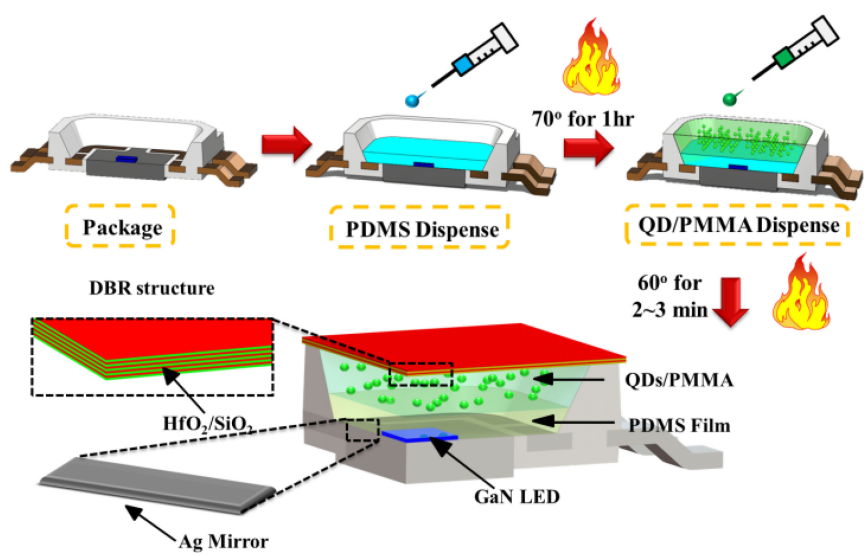

Fig. 1. Schematic flow chart of cross-sectional view DBR embedded remote QD dispense structure.

centered at $400 \mathrm{~nm}$ and a full width of approximately $60 \mathrm{~nm}$ was employed to enhance the utilization of UV light for producing a higher output intensity. Therefore, the QD-WLEDs demonstrated high CRI and excellent angular CCT ranging from 2500 to $4500 \mathrm{~K}$.

\section{EXPERIMENT}

Fig. 1 presents a cross-sectional view of a remote QDdispensing device with a DBR structure. For the conventional dispensing structure, the PMMA was mixed with the QDs to form a QD/PMMA blend [30]. The advantage of PMMA is that its solidifying temperature is lower than that of silicone and epoxy, which can prevent QD degradation during the curing step of the encapsulant. Furthermore, the PMMA can be readily dissolved in chloroform or chlorobenzene, which is commonly used to disperse QDs because of the concern regarding the surface of the capped organic ligands [31], [32]. Because the PMMA is compatible with QD solutions, a highly homogeneous blending of the two chemicals can be realized, and the subsequent self-clustering should be reduced. The QD-LEDs were fabricated using the following steps: 1) The LED package was first half-filled with polydimethylsiloxane (PDMS). 2) The QD/PMMA blend was mixed at a volume ratio of $2: 1$ and dispensed in the package to fill the remaining space in the package. 3) The QD-LED was baked at $60^{\circ} \mathrm{C}$ for $2-3 \mathrm{~min}$, and the DBR structure was finally placed on the surface of the package.

In the finished package, several vital components must be selected carefully to enhance the overall performance. First, the appropriate LED chip was selected. A $1000 \times 1000 \mu \mathrm{m}$ UV LED with a $380 \mathrm{~nm}$ emission was used because of the high luminescent efficiency of the QDs under UV excitation [33], [34]. Second, the DBR structure was used to increase the utilization of high energy photons by reflecting them back onto the QD layer. Therefore, $\mathrm{HfO}_{2}$ and $\mathrm{SiO}_{2}$ were employed as the dielectric materials in the DBR structure. Third, the ratios between different colors of QDs, which can affect the final CCT considerably, were determined. Table I shows the different CCT output values. Fourth, the PDMS was used to provide heat isolation to the QD layer and prevent the QD/PMMA from sifting through and reaching the LED chip. Silicone is the most frequently used LED filling materials; however, because of its
TABLE I

THE MiXING Volume RATIO OF RGB QDS FOR WLEDS WITH DIFFERENT COLOR TEMPERATURES

\begin{tabular}{lccc}
\hline \hline CCT & RED & Green & Blue \\
\hline $2500 \mathrm{~K}$ & 1 & 240 & 200 \\
$3500 \mathrm{~K}$ & 1 & 400 & 300 \\
$4500 \mathrm{~K}$ & 1 & 500 & 700 \\
\hline \hline
\end{tabular}

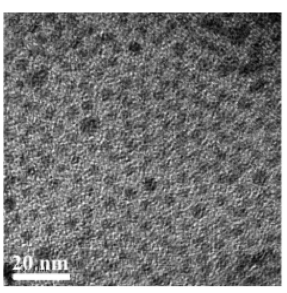

(a)

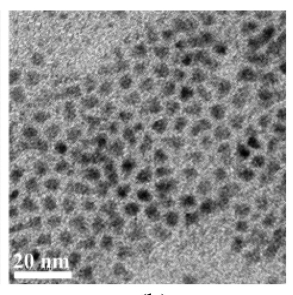

(b)

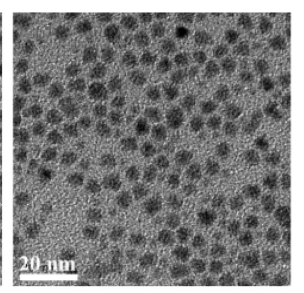

(c)
Fig. 2. TEM images of QDs with the emission colors of (a) blue, (b) green, and (c) red.

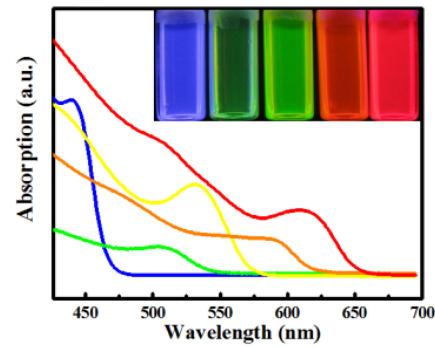

(a)

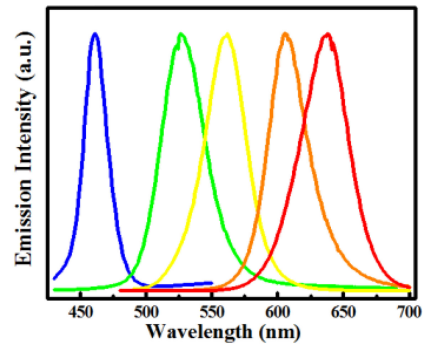

(b)
Fig. 3. (a) Absorption spectra and (b) emission spectra of blue, green, yellow, orange, and red QDs.

chemical reactivity with chlorobenzene in QD solutions, it is not suitable for this device.

\section{RESULT AND DISCUSSION}

Fig. 2 shows the transmission electron microscope (TEM) images of blue, green, and red QDs. The average diameters of the blue, green, and red QD samples are estimated as $3.58 \pm$ $0.22,4.16 \pm 0.21$, and $5.41 \pm 0.34 \mathrm{~nm}$, respectively; these QD samples are spherical and their sizes are uniform. Fig. 3 shows the UV-visible absorption and photoluminescence (PL) spectra of five QD products (Sigma-Aldrich Company) used in this experiment. The wavelength of peak emission of the CdS QDs is located at $460 \mathrm{~nm}$ and that of the $\mathrm{CdSe} / \mathrm{ZnS}$ QDs is located at 530, 560, 610, and $650 \mathrm{~nm}$; the full-widths at half-maximum of these products are approximately 20, 40, 40, 35, and $40 \mathrm{~nm}$, respectively.

Fig. 4(a) and (b) illustrate the measured reflectivity and absorption spectra of the DBR structure comprising 11 pairs of $\mathrm{HfO}_{2} / \mathrm{SiO}_{2}$. The DBR structure was produced using an ionassisted e-gun system to evaporate the 11 pairs of $\mathrm{HfO}_{2} / \mathrm{SiO}_{2}$. The refractive indices of the $\mathrm{HfO}_{2}$ and $\mathrm{SiO}_{2}$ layers are 1.9 and 1.46 , respectively. The maximum reflectivity was designed for a central wavelength of $400 \mathrm{~nm}$, and more than $90 \%$ of the 


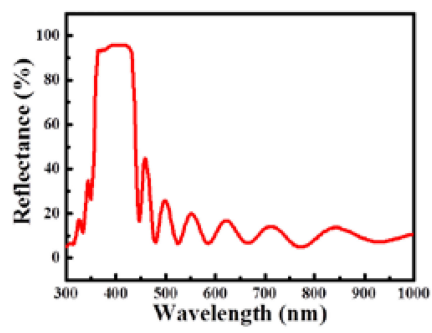

(a)

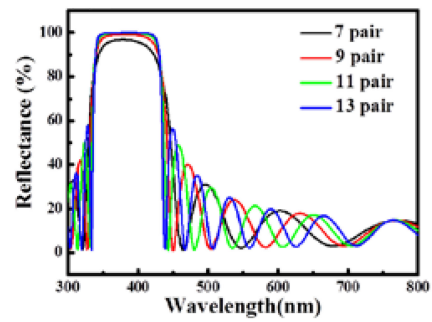

(c)

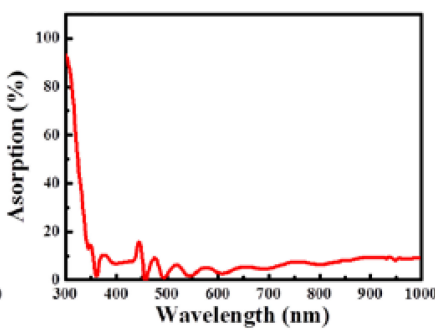

(b)

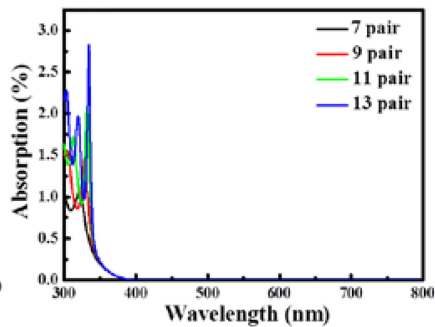

(d)

Fig. 4. The measured (a) reflectivity and (b) absorption spectra of 11 pairs of $\mathrm{HfO}_{2} / \mathrm{SiO}_{2}$ DBR structures with wavelengths ranging from 300 to $800 \mathrm{~nm}$. The simulated (c) reflectance and (d) absorption spectra of different pairs of $\mathrm{HfO}_{2} / \mathrm{SiO}_{2} \mathrm{DBR}$ structures with wavelength ranging from 300 to $800 \mathrm{~nm}$.

reflectivity was maintained between 365 and $430 \mathrm{~nm}$, which is sufficient for the wavelength of the InGaN UV-LED $(\lambda=$ $380 \mathrm{~nm}$ ) in the experiment conducted in this study. The reflectivity is relatively low in the visible spectral range, which enabled the transmission of visible photons produced by the QDs. The absorption loss is approximately $10 \%$ at $380 \mathrm{~nm}$, and less than $10 \%$ between 450 and $700 \mathrm{~nm}$. This result indicates that the DBR structure is ideal for use in the WLED. Fig. 4(c) and (d) depicts the simulated reflection and absorption spectra (for the wavelength range of $300-800 \mathrm{~nm}$ ) of the DBR structure containing different pairs of $\mathrm{HfO}_{2} / \mathrm{SiO}_{2}$. Although the reflectivity increases as we increase the pair number, a sharp rise of absorption for 13 pairs of DBR indicates the upper limit of pair number shall be between 11 and 13 . Thus the choice of 11 pairs can be conservative but safe to avoid extra absorption loss at UV wavelength.

Fig. 5 shows the blue, green, yellow, orange, and red monochromatic QD-LEDs at a driving current of $150 \mathrm{~mA}$ with peak wavelengths of 460, 530, 560, 610, and $640 \mathrm{~nm}$, respectively. The results demonstrate the outstanding characteristics of the QDs, such as narrow bandwidth and size-tunable bandgap, as well as the substantial enhancement after capping the DBR structure. Additional evaluations indicated that the power intensity enhancement ratio between the samples with and without DBR for the blue, green, yellow, orange and red QD-LEDs are $14.6 \%, 37.8 \%, 38.9 \%, 29.4 \%$, and $39.4 \%$, respectively. Furthermore, the $30 \mathrm{~nm}$ emission bandwidth of the QDs can yield a high degree of color purity compared with the estimated $100 \mathrm{~nm}$ bandwidth of the monochromatic phosphor-converted LEDs [35], [36]. Fig. 5 also shows the photographs of the red, green, blue, yellow, and orange monochromatic QD-LEDs operated at $100 \mathrm{~mA}$. The peak wavelength of the monochromatic QD-LEDs demonstrates a slight red shift and broadened bandwidth, which can be attributed to the CdSe QD aggregation inside the PMMA resin [37].

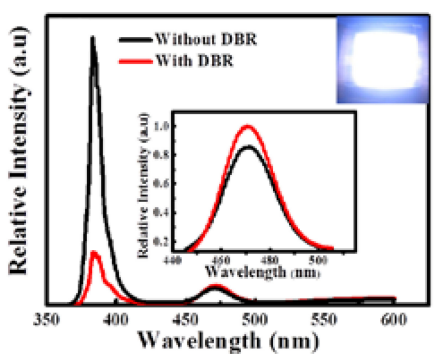

(a)

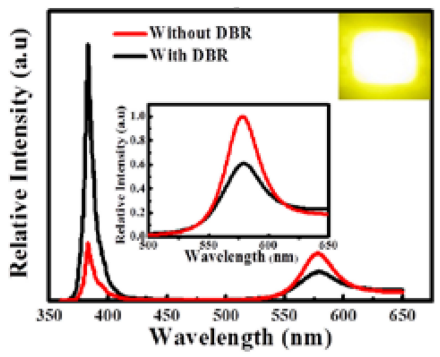

(c)

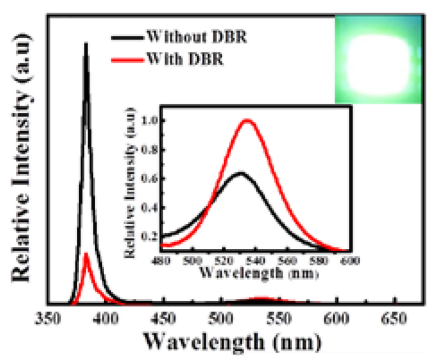

(b)

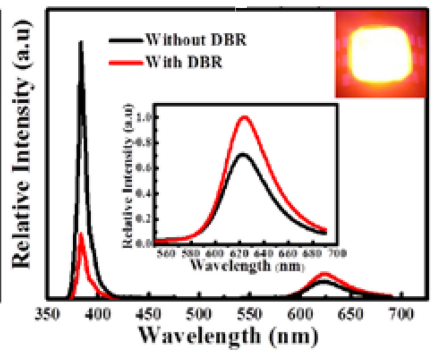

(d)

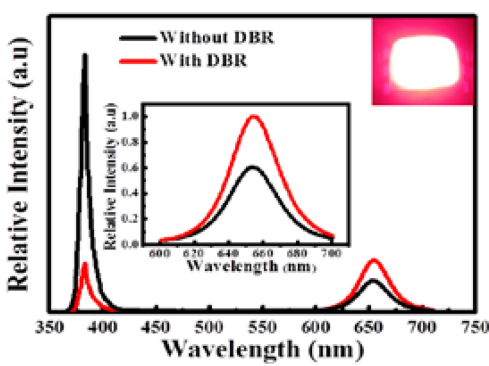

(e)

Fig. 5. (a)-(e) Relative emission spectra of monochromatic QDs with and without the DBR structure at $150 \mathrm{~mA}$, and the insets indicate the images of the device with the DBR structure.

Apart from single color devices, appropriately mixed QDs can generate white light at different CCTs. WLEDs demonstrating three CCTs with and without DBR can be created using the ratios listed in Table I. Moreover, the intensity of red light clearly demonstrates a relatively high enhancement after capping the DBR. This can be attributed to reabsorption of green and blue light as well as the substantially high stability of the red QDs. The CIE color coordinates of the QD-WLEDs with DBR for three CCTs are $(0.35,0.33),(0.40,0.41)$, and $(0.44,0.37)$, as shown in Fig. 6(d)-(f). In particular, the CIE color coordinates of the $4500 \mathrm{~K}$ WLED are similar to the Planckian locus, and the coordinates of the other CCT WLEDs are also consistent with the trend of the Planckian locus. When this trichromatic QD-LED method is used, the WLEDs can render light similar to natural sunlight at various CCTs and have the potential to replace traditional lighting devices.

The luminous efficiency of the LED device with the DBR structure and containing a UV LED source is approximately $8.4 \mathrm{~lm} / \mathrm{W}$, and this is consistent with the results of previous studies [38]-[40]. This improvement can be attributed to the enhancement of the UV excitation between the DBR structure and the highly reflective silver mirror at the bottom of the LED package, which excites the RGB QD and derives a higher intensity. In addition, Fig. 7(b) depicts the current-dependent CRI. 


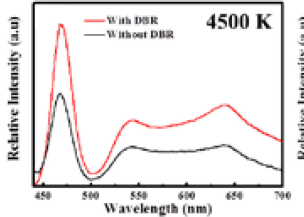

(a)

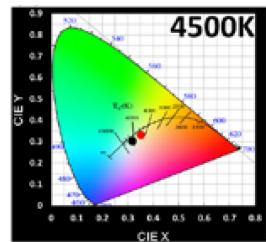

(d)

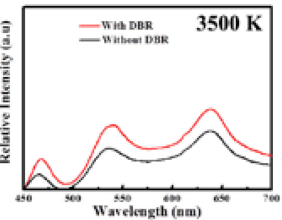

(b)

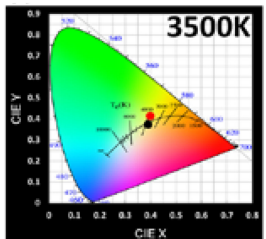

(e)

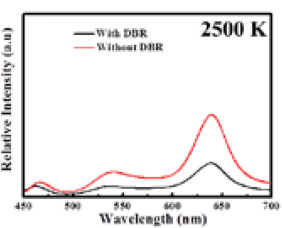

(c)

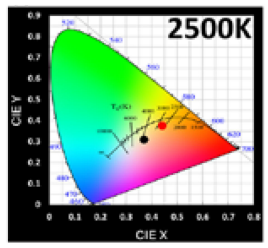

(f)
Fig. 6. (a)-(c) Emission spectra and (d)-(f) CIE chromaticity coordinate of RGB QD-WLED at CCTs of 4500, 3500, and $2500 \mathrm{~K}$ with and without the $\mathrm{DBR}$ at $150 \mathrm{~mA}$.

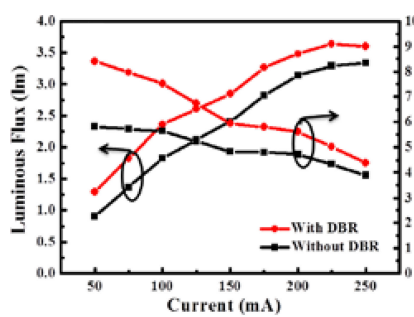

(a)

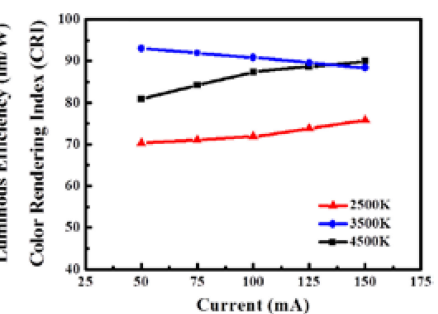

(b)
Fig. 7. Current-dependent (a) lumen and luminous efficiency of LED the device with and without the DBR structure at $3500 \mathrm{~K}$ (b) CRI of the RGB QD -WLEDs at various CCTs.

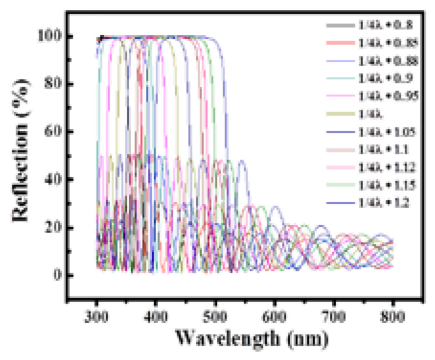

(a)

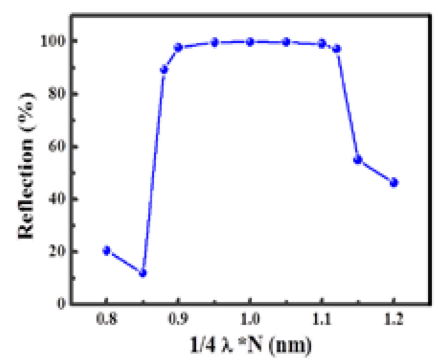

(b)
Fig. 8. (a) Simulated reflection spectra at wavelengths ranging from 300 to $800 \mathrm{~nm}$. (b) The calculated reflectivity at 380nm with various DBR thickness conditions.

The 3500 and $4500 \mathrm{~K}$ samples register a CRI of over 90, which is prominent for indoor lighting. The CRI of the $2500 \mathrm{~K}$ WLEDs is low because the intensity of red light is substantially higher than that of the blue and green components. Therefore, the CRI can be improved further by fine-tuning the mixing ratio of the RGB QDs to ensure the uniformity of the relative intensities of the RGB components.

Fig. 8 shows the simulated reflection spectra at wavelengths ranging from 300 to $800 \mathrm{~nm}$ as well as the reflection at $380 \mathrm{~nm}$. The reflectivity at $380 \mathrm{~nm}$ is focused here because it can be linearly correlated towards the enhancement of visible light emis-

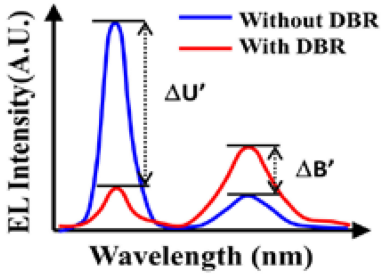

(a)

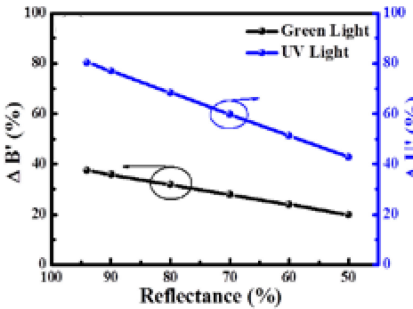

(c)

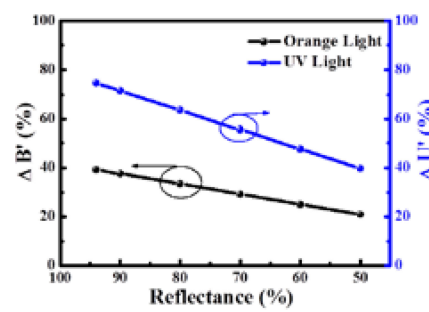

(e)

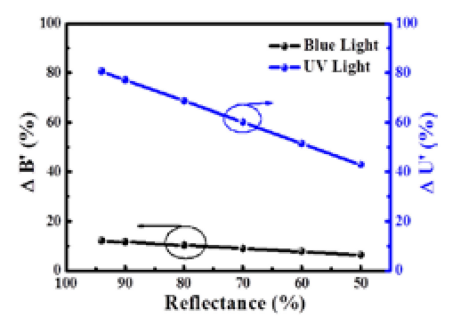

(b)

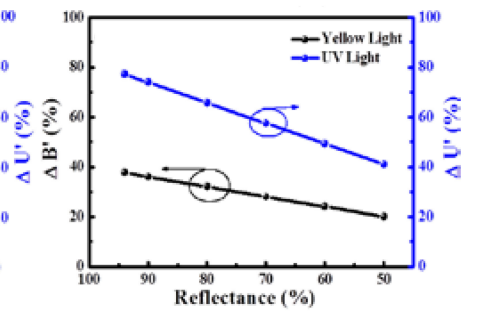

(d)

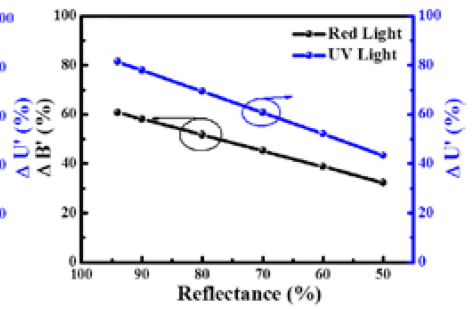

(f)
Fig. 9. (a) The illustration of the intensity spectrum for hybrid QD LED with and without DBR structure. (b)-(f) illustrate the plots of $\Delta U^{\prime}$ for UV light reduction and $\Delta \mathrm{B}^{\prime}$ for the enhancement of blue, green, yellow, orange, and red monochromatic QD-LEDs. All the calculation is based on the linear extrapolation of the measured results.

sion. Under the same quantum yield of CQDs, more reflection on UV photons means more emission of visible ones.

In Fig. 8(b), a variation of $2 \%$ reflectivity at $380 \mathrm{~nm}$ can be maintained within the regular $\pm 10 \%$ thickness variation. Only when the layer changed over $15 \%$ of thickness, the reflectivity drops sharply to $40 \%$ of the original values. So from this simulation, we can conclude that a change of $10 \%$ in DBR structure is tolerable in our device.

As for the influences on the device performance, we could use a simple linear model to obtain the first order estimation. Shown in Fig. 9, compare the spectra with and without DBR, the amount of UV reduction ( $\Delta \mathrm{U}^{\prime}$ ) can be correlated to enhancement of visible $\left(\Delta \mathrm{B}^{\prime}\right)$. This $\Delta \mathrm{U}^{\prime}$ changes linearly with the reflectivity at $380 \mathrm{~nm}$, and when reflectivity becomes zero, no enhancement can be detected. So a linear fit for various colors can be demonstrated in Fig. 9(b)-(f), according to various reflectivities at $380 \mathrm{~nm}$. To set a standard of $90 \%$ of the current enhancement, the reflectivity cannot be lower than $85 \%$, which also comply with our previous $\pm 10 \%$ DBR fabrication tolerance.

To evaluate the light quality of three CCT WLEDs further, this study analyzed the deviation of the angular CCT. The deviation of the angular CCT can be determined by subtracting the minimum CCT from the maximum CCT in the range between $-70^{\circ}$ and $70^{\circ}$. Fig. 10 depicts the distribution of the angular CCT of the QD-WLED sample at 2500, 3500, and $4500 \mathrm{~K}$; the deviation of the angular CCT in the WLEDs can be improved considerably 


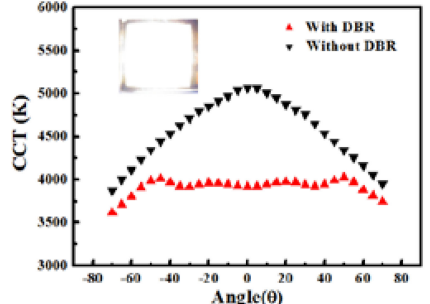

(a)

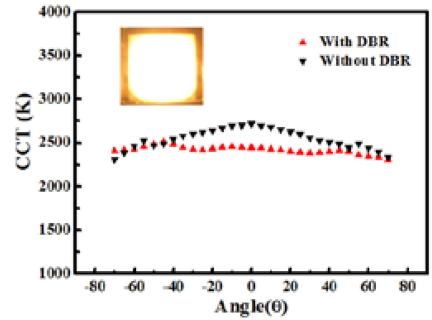

(c)

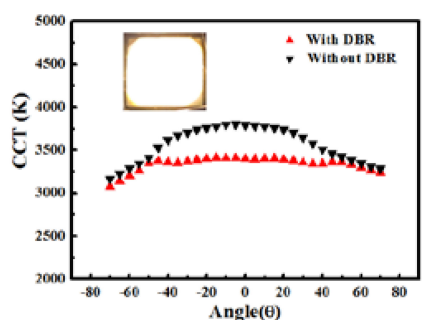

(b)

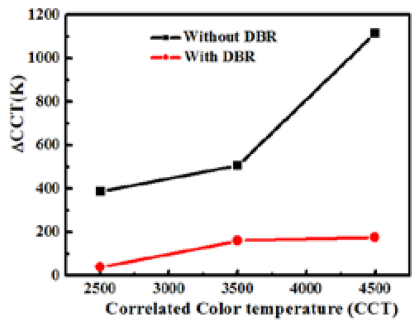

(d)
Fig. 10. Angular-dependent CCT with and without DBR of (a) $4500 \mathrm{~K}$, (b) $3500 \mathrm{~K}$, and (c) $2500 \mathrm{~K}$. (d) The CCT deviation for three samples with and without DBR.

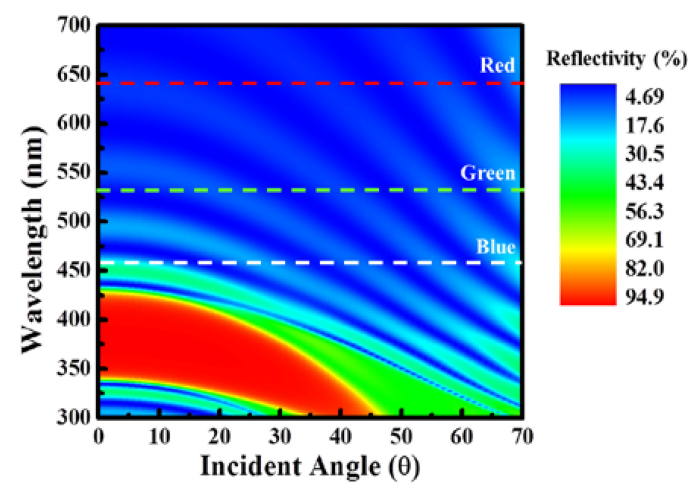

Fig. 11. Color mapping plot of the reflectivity of the DBR at the various incident angles.

by capping the top of the package with a DBR. The CCT deviations of the QD-WLEDs with and without DBR at 4500 and $3500 \mathrm{~K}$ improved from 1114 and $621 \mathrm{~K}$ to 177 and $160 \mathrm{~K}$.

Specifically, the CCT of the QD-WLEDs with the DBR structure at $2500 \mathrm{~K}$ decreased to $39 \mathrm{~K}$, which indicates excellent color uniformity at various angles. With such uniformity, unwanted phenomena, such as yellow rings, can be avoided. In general, the conventional WLED structure has an inferior angular CCT deviation because of the poor extraction of blue light at wide angles. With DBR structure, as highlighted in the following paragraph, the ratio of blue over other colors can be appropriately adjusted such that blue photons are suppressed in the normal direction and the CCT uniformity is improved.

To examine the variation of the DBR reflection and its effect on the CCT uniformity improvement, this study used the TFcalc simulation software to analyze the characteristics of the DBR structure. Fig. 11 shows the reflectivity of the DBR structure at various angles. The reflectivity of the DBR structure in the normal direction demonstrates an almost total reflection of the
UV light, and the reflection of the blue light region is higher than that of the green and red light regions. Therefore, the CCT can be suppressed in the normal direction because of the lower transmittance of blue light. In addition, the reflectivity of the DBR structure at the peak wavelength of the UV chip (380 nm) considerably decreased by $40 \%$ when the incident angle exceeded $35^{\circ}$. Moreover, the reflectivity of the blue light region also decreased by the same amount as the green and red light regions when the incident angle exceeded $35^{\circ}$. The simulated results indicate that the short wavelength reflectivity of DBR at an angle greater than $35^{\circ}$ can be suppressed, and more blue photons are permitted at wide angles than in the normal direction. This reduction in reflectivity can change the ratio of the blue to yellow photons and thus modify the CCT at wide angles. Furthermore, the extra cost due to the addition of DBR structure can be estimated as only $0.2 \%$ of the overall QD-WLED package budget. Therefore, the technology of QD-WLEDs with DBR can provide light sources with high CRIs and uniform CCTs, which can potentially replace traditional lighting in the next generation of SSL.

\section{CONCLUSION}

This study presents DBR capped monochromatic QDWLEDs with various CCTs that can be possible candidates for the future generation of SSL. The DBR capped onto the top of the package can increase the intensity of the QD emission by reflecting the UV light for both monochromatic QD-LEDs and QD-WLEDs. Moreover, the CRI of the WLED is greater than 90. Therefore, the CCT angular deviation of the QD-WLED can decrease to $39 \mathrm{~K}$ because of the reflectivity variation of the DBR at various incident angles, which is an attractive approach for high quality lighting applications.

\section{ACKNOWLEDGMENT}

The authors would like to thank the Helio Opto Corporation for providing technical support, Dr. C.-F. Chu in the SemiLEDs Corporation and Dr. C.-H. Chiu for their suggestions in Advanced Optoelectronic Technology Inc., Hsinchu, Taiwan.

\section{REFERENCES}

[1] E. F. Schubert and J. K. Kim, "Solid-state light sources getting smart," Science, vol. 308, pp. 1274-1278, 2005

[2] S. Coe, W. K. Woo, M. Bawendi, and V. Bulovic, "Electroluminescence from single monolayers of nanocrystals in molecular organic devices," Nature, vol. 420, pp. 800-803, 2002.

[3] K. J. Chen et al., "White light emitting diodes with enhanced CCT uniformity and luminous flux using $\mathrm{ZrO}_{2}$ nanoparticles," Nanoscale, vol. 6 , pp. 5378-5383, 2014.

[4] N. Kimura et al., "Extrahigh color rendering white light-emitting diode lamps using oxynitride and nitride phosphors excited by blue lightemitting diode," Appl. Phys. Lett., vol. 90, p. 051109, 2007.

[5] S. Nakamura, T. Mukai, and M. Senoh, "Candela-class high-brightness ingan/algan double-heterostructure blue-light-emitting diodes," Appl. Phys. Lett., vol. 64, pp. 1687-1689, 1994.

[6] H. C. Chen et al., "A novel randomly textured phosphor structure for highly efficient white light-emitting diodes," Nanoscale Res. Lett., vol. 7, pp. 1-5, 2012.

[7] M. R. Krames et al., "Status and future of high-power light-emitting diodes for solid-state lighting," J. Disp. Technol., vol. 3, pp. 160-175, 2007. 
[8] H. C. Kuo et al., "Patterned structure of remote phosphor for phosphorconverted white LEDs," Opt. Exp., vol. 19, pp. A930-A936, 2011.

[9] H. C. Chen et al., "Improvement in uniformity of emission by $\mathrm{ZrO}_{2}$ nano-particles for white LEDs," Nanotechnology, vol. 23, pp. 2652010126520105, 2012.

[10] S. E. Brinkley et al., "Robust thermal performance of Sr2Si5N8:Eu2+: An efficient red emitting phosphor for light emitting diode based white lighting," Appl. Phys. Lett., vol. 99, p. 241106, 2011.

[11] K. J. Chen et al., "High thermal stability of correlated color temperature using current compensation in hybrid warm white high-voltage LEDs," Opt. Exp., vol. 21, pp. A201-A207, 2013.

[12] C. W. Yeh et al., "Origin of thermal degradation of $\mathrm{Sr}_{2-\mathrm{x}} \mathrm{Si}_{5} \mathrm{~N}_{8}: \mathrm{Eu}_{\mathrm{x}}$ phosphors in air for light-emitting diodes," J. Amer. Chem. Soc., vol. 134 pp. 14108-14117, 2012

[13] A. A. Setlur et al., "Energy-efficient, high-color-rendering LED lamps using oxyfluoride and fluoride phosphors," Chem. Mater, vol. 22, pp. 4076-4082, 2010.

[14] K. J. Chen et al., "Enhanced luminous efficiency of WLEDs using a duallayer structure of the remote phosphor package," J. Disp. Technol., vol. 9, pp. 280-284, 2013.

[15] P. Reiss, J. Bleuse, and A. Pron, "Highly luminescent CdSe/ZnSe core/shell nanocrystals of low size dispersion," Nano Lett., vol. 2, pp. 781-784, 2002.

[16] I. L. Medintz, H. T. Uyeda, E. R. Goldman, and H. Mattoussi, "Quantum dot bioconjugates for imaging, labelling and sensing," Nature Mater. vol. 4, pp. 435-446, 2005.

[17] Y. Shirasaki, G. J. Supran, M. G. Bawendi, and V. Bulovic, "Emergence of colloidal quantum-dot light-emitting technologies," Nature Photon., vol. 7, pp. 13-23, 2013.

[18] P. Gu et al., "Real-time and on-chip surface temperature sensing of GaN LED chips using PbSe quantum dots.," Nanoscale, vol. 5, pp. 1048110486, 2013.

[19] E. Jang et al., "White-light-emitting diodes with quantum dot color converters for display backlights," Adv. Mater, vol. 22, pp. 3076-3080, 2010.

[20] F. Ren, H. Zhao, F. Vetrone, and D. Ma, "Microwave-assisted cation exchange toward synthesis of near-infrared emitting $\mathrm{PbS} / \mathrm{CdS}$ core/shell quantum dots with significantly improved quantum yields through a uniform growth path," Nanoscale, vol. 5, pp. 7800-7804, 2013.

[21] T. H. Kim et al., "Full-colour quantum dot displays fabricated by transfer printing." Nature Photon., vol. 5, pp. 176-182, 2011.

[22] T. Zhu et al., "Mist fabrication of light emitting diodes with colloidal nanocrystal quantum dots," Appl. Phys. Lett., vol. 92, p. 023111, 2008.

[23] V. Wood et al., "Inkjet-printed quantum dot-polymer composites for fullcolor AC-driven displays," Adv. Mater, vol. 21, pp. 2151-2155, 2009.

[24] Y. C. Pu and Y. J. Hsu, "Multicolored Cd(1-x)Zn(x)Se quantum dots with type-I core/shell structure: Single-step synthesis and their use as light emitting diodes," Nanoscale, vol. 6, pp. 3881-3888, 2014.

[25] C. Dang et al., "A wafer-level integrated white-light-emitting diode incorporating colloidal quantum dots as a nanocomposite luminescent material," Adv. Mater., vol. 24, pp. 5915-5918, 2012.

[26] K. J. Chen et al., "Resonant-enhanced full-color emission of quantumdot-based display technology using a pulsed spray method," Adv. Funct. Mater., vol. 22, pp. 5138-5143, 2012.

[27] H. S. Chen, C. K. Hsu, and H. Y. Hong, "InGaN-CdSe-ZnSe quantum dots white LEDs," IEEE Photon. Technol. Lett., vol. 18, no. 1, pp. 193-195, Jan. 2006.

[28] H. S. Jang et al., "White light-emitting diodes with excellent color rendering based on organically capped CdSe quantum dots and $\mathrm{Sr}_{3} \mathrm{SiO}_{5}: \mathrm{Ce}^{3+}$, $\mathrm{Li}^{+}$phosphors," Adv. Mater, vol. 20, pp. 2696-2702, 2008.

[29] S. Nizamoglu, G. Zengin, and H. V. Demir, "Color-converting combinations of nanocrystal emitters for warm-white light generation with high color rendering index," Appl. Phys. Lett., vol. 92, p. 031102, 2008.

[30] H. Song and S. Lee, "Photoluminescent (CdSe) ZnS quantum dotpolymethylmethacrylate polymer composite thin films in the visible spectral range," Nanotechnology, vol. 18, pp. 05540201-05540206, 2007.

[31] W. S. Song, H. J. Kim, Y. S. Kim, and H. Yang, "Synthesis of Ba2Si3O8:Eu2 + Phosphor for fabrication of white light-emitting diodes assisted by ZnCdSe/ZnSe quantum dot," J. Electrochem. Soc. vol. 157, pp. J319-J323, 201.

[32] K. J. Chen et al., "The Influence of the thermal effect on $\mathrm{CdSe} / \mathrm{ZnS}$ quantum dots in light-emitting diodes," J. Lightw. Technol. vol. 30, no. 14, pp. 2256-2261, Jul. 2012

[33] P. M. Tu et al., “"'Investigation of efficiency droop for InGaN-based UV light-emitting diodes with InAlGaN barrier," Appl. Phys. Lett., vol. 98, p. 211107, 2011.
[34] C. F. Lai et al., "Far-field and near-field distribution of gan-based photonic crystal leds with guided mode extraction," IEEE J. Sel. Topics Quantum Electron., vol. 15, no. 4, pp. 1234-1241, Jul./Aug. 2009.

[35] J. R. Oh et al., "Full down-conversion of amber-emitting phosphorconverted light-emitting diodes with powder phosphors and a long-wave pass filter," Opt. Exp., vol. 18, pp. 11063-11072, 2010.

[36] J. H. Oh, J. R. Oh, H. K. Park, Y.-G. Sung, and Y. R. Do, "Highly-efficient, tunable green, phosphor-converted LEDs using a long-pass dichroic filter and a series of orthosilicate phosphors for tri-color white LEDs," Opt. Exp., vol. 20, pp. A1-A12, 2012.

[37] J. Lim et al., "Preparation of highly luminescent nanocrystals and their application to light-emitting diodes," Adv. Mater., vol. 19, pp. 1927-1932, 2007.

[38] H. S. Chen, K. W. Wang, S. S. Chen, and S. R. Chung, " $\mathrm{Zn}_{\mathrm{x}} \mathrm{Cd}_{1-\mathrm{x}} \mathrm{S}$ quantum dots-based white light-emitting diodes," Opt. Lett., vol. 38, pp. 2080-2082, 2013.

[39] H. Wang, K. S. Lee, J. H. Ryu, C. H. Hong, and Y. H. Cho, "White light emitting diodes realized by using an active packaging method with $\mathrm{CdSe} / \mathrm{ZnS}$ quantum dots dispersed in photosensitive epoxy resins" Nanotechnology, vol. 19, pp. 14520201-14520204, 2008.

[40] T. Nguyen Ngoc, L. Quynh-Phuong, S. Bui Thanh, S. Le Hoang, and J.-Y. Bae, "Enhanced fluorescence, morphological and thermal properties of $\mathrm{CdSe} / \mathrm{ZnS}$ quantum dots incorporated in silicone resin," J. Nanosci. Nanotechnol., vol. 13, pp. 434-442, 2013.

Kuo-Ju Chen was born in Taichung, Taiwan. He received the B.S. degree in industry education from National Kaohsiung Normal University, Kaohsiung, Taiwan, in 2008, and the M.S. degree from National Taiwan Normal University. $\mathrm{He}$ is currently working toward the Ph.D. degree at the National Chiao-Tung University, Hsinchu, Taiwan.

His study focusses on UV excitable phosphate, which has high-luminous efficiency and high stability. He used combinatorial chemistry to develop UVexcited phosphor for his master thesis. His Ph.D. research includes fabrication, simulation, and characterization for high-power light-emitted diodes.

Chien-Chung Lin (S'93-M'02-SM'13) was born in Taipei, Taiwan, in 1970. He received the B.S. degree in electrical engineering from the National Taiwan University, Taipei, in 1993, and the M.S. and Ph.D. degrees in electrical engineering from Stanford University, Stanford, CA, USA, in 1997 and 2002, respectively. His thesis work focused on design, modeling, and fabrication of micromachined tunable optoelectronic devices. Since 2009, he has been with National Chiao-Tung University (NCTU) in Tainan, Taiwan, where he holds a position as an Associate Professor. The major research efforts in his group are in design and fabrication of semiconductor optoelectronic devices, including LEDs, solar cells, and lasers. Before joining NCTU, he worked for different start-ups in the United States. After graduating from Stanford in 2002, he joined E2O Communications, Inc., in Calabasas, CA, USA, as a Senior Optoelectronic Engineer. His main research interests then were in optically and electrically pumped long-wavelength vertical cavity surface-emitting lasers. In 2004, he joined Santur Corporation, Fremont, CA, where he initially worked as a Member of technical staff then became a Manager of Laser Chip Engineering later. He had worked on various projects such as monolithic multiwavelength DFB laser arrays for data and telecommunications applications, yield and reliability analysis of DFB Laser arrays, etc. He has more than 60 journal and conference publications and is a Senior Member of the IEEE Photonic Society and Electron Devices Society.

Hau-Vei Han born in Hsinchu, Taiwan, in 1986. He received the B.E. degree from National Chiao Tung University (NCTU), Hsinchu, Taiwan, in 2009, and the M.S. degree from the Institute of Photonic System, NCTU, in 2011, where he is currently working toward the Ph.D. degree in the Institute of ElectroOptical Engineering. His research interests include nanostructure analysis for solar cells, FDTD's simulation for optical devices, and quantum dots hybrid solar cells under the instruction of Prof. H.-C. Kuo, Prof. P. Yu, and Prof. C.-C. Lin. 
Chia-Yu Lee was born in Chiayi, Taiwan. He received the M.S. degree from the Department of Optics and Photonics, National Central University, Taoyuan, Taiwan. He is currently working toward the Ph.D. degree in the Institute of Electro-Optical Engineering, National Chiao Tung University, Taiwan. His research interests include epitaxy growth by metal-organic chemical-vapor deposition for light-emitting diodes and analyzing the optical and electrical characteristic

Shih-Hsuan Chien was born in Taoyuan, Taiwan. He received the B.S. degree from National Chiao-Tung University (NCTU), Hsinchu, Taiwan, in 2012, where he is currently working toward the M.S. degree. His master's focus is on high-powered white light-emitting diodes, including fabrication, simulation, measurement, and optical characteristic study. In addition, he researches optical applications of a promising material quantum dots.

Kuan-Yu Wang born in Taipei, Taiwan, in 1990. He received the B.E. degree from National Chung Cheng University, Chiayi, Taiwan, in 2012. He is currently working toward the M.S. degree in the College of Photonic, National ChiaoTung University, Taiwan. His research interests include LED package, optics work's simulation for optical devices, and quantum dots white light-emitting diode under the instruction of Prof. H.-C. Kuo and Prof. C.-C. Lin.

Sheng-Huan Chiu was born in Taichung, Taiwan. He received the B.S. degree from National Chiao-Tung University, Hsinchu, Taiwan, in 2012, where he is currently working toward the M.S. degree. His master's degree focus is on high-powered white light-emitting diodes, including fabrication, simulation, measurement, and optical characteristic study. In addition, he researches optical applications of a promising material quantum dots.

Zong-Yi Tu was born in Tainan, Taiwan. He received the B.S. degree from the Department of Electronic and Computer Engineering, National Taiwan University of Science and Technology (NTUST), Taipei, Taiwan, in 2013. He is currently pursuing the M.S. degree at National Chiao-Tung University (NCTU), Hsinchu, Taiwan. His master's degree focus is on high luminous efficiency and high CRI white light-emitting diodes, including fabrication, simulation, measurement, and optical characteristic study. In addition, he researches optical applications of a promising material quantum dot.

Jie-Ru Li was born in Hsinchu, Taiwan. She received the B.S. degree from National Changhua University of Education (NCUE), Changhua, Taiwan, in 2013. She is now a master's student at National Chaio-Tung University (NCTU), Hsinchu. Her master's focus is on quantum dot-based white light for LEDbacklights and white light-emitting diodes, including fabrication, simulation, measurement, and optical characteristic study.
Teng-Ming Chen received the B.S. degree in chemistry from the National Tsing-Hwa University, Taiwan, in 1977, and the Ph.D. degree in solid-state chemistry from Iowa State University, Ames, IA, USA, in 1986. From 1981 to 1984, he served as a Graduate Research Assistant at the Ames Laboratory of the Department of Energy. He became a Postdoctoral Fellow of the Chemistry Department, University of Virginia, Charlottesville, VA, USA, from 1986 to 1988. He then joined the Department of Applied Chemistry, National Chiao Tung University, Taiwan, as an Associate Professor from 1988 to 1994. He is currently a Professor in the same department and serves as a Consultant for the Energy and Resources Laboratories of ITRI. His current research interests include materials chemistry of inorganic phosphors, synthesis and applications of luminescent semiconductor quantum dots and magnetic nanoparticles, and phosphorescent dopants for OLEDs and device fabrications. He served as a Quest Scientific Reviewer for international journals such as the Journal of the American Chemical Society, Chemistry of Materials, Journal of Solid-State Chemistry, Journal of Luminescence, and Materials Chemistry and Physics. In addition, he has also has served on the editorial board of the Chinese Journal of Luminescence since 2004.

Xiuling Li received the B.S. degree in physics chemistry from Peking University, Haidian, China, in 1986, and the Ph.D. degree in physics chemistry from the University of California, Los Angeles, CA, USA, in 1993. She is currently an Associate Professor of electrical and computer engineering, University of Illinois, Urbana -Champaign, IL, USA. Her research interests include the area of semiconductor materials and devices, which are focused on the following projects: 1) the growth and fabrication of GaAs, InP, and GaN-based III-V compound semiconductor 3-D structures at the nanometer scale, and the realization of 3-D semiconductor optoelectronic devices for potential applications in chemical and biological sensing; 2) the development of high-efficiency GaAs-based terahertz photomixer and the on-chip integration with heterodyned tunable lasers and waveguide; 3) InP-based high-power lasers for applications as pump sources for fiber lasers; and 4) III-V semiconductor nanowires/nanorods formation through engineered top-down approach and applications toward nanoinjection lasers. She has authored more than 100 journal and conference publications.

Min-Hsiung Shih received the B.S. degree in physics from the National Cheng Kung University, Tainan, Taiwan, in 1995, the M.S. degree in physics from the National Tsing Hua University, Taiwan, in 1997, and the Ph.D. degree in electrical engineering/electrophysics from the University of Southern California, Los Angeles, CA, USA, in 2006

He is currently an Associate Research Fellow in the Research Center for Applied Sciences, Academia Sinica, Nankang, Taiwan. He has authored more than 50 journal and conference publications. His research interests include integrated photonic circuits, photonic crystals, GaN-based lasers, surface plasmonics, and cavity quantum electrodynamics.

Hao-Chung Kuo (S'98-M'99-SM'06-F'15) received the B.S. degree in physics from National Taiwan University, Taiwan, the M.S. degree in electrical and computer engineering from Rutgers University, New Brunswick, NJ, USA, in 1995, and the Ph.D. degree from the University of Illinois, Urbana Champaign, IL, USA, in 1999. He has an extensive professional career both in research and industrial research institutions that includes: Research Assistan in Lucent Technologies, Bell Laboratories from 1993 to 1995; and a Senior R\&D Engineer in Fiber Optics Division at Agilent Technologies from 1999 to 2001, and LuxNet Corporation from 2001 to 2002. Since October 2002, he has been with the National Chiao Tung University (NCTU), Hsinchu, Taiwan, as a Faculty Member of the Institute of Electro-Optical Engineering. He is currently the Associate Dean, Office of International Affair, NCTU. His current research interests include semiconductor lasers, VCSELs, blue and UV LED lasers, quantum-confined optoelectronic structures, optoelectronic materials, and solar cells. He has authored and coauthored 300 internal journal papers, two invited book chapter, and six granted and 12 pending patents. He is the Associate Editor of the IEEE/OSA JOURNAL OF LIGHTWAVE TECHNOLOGY and a Guest Editor of the IEEE JOURNAL OF SELECTED TOPICS IN QUANTUM ELECTRONICS issue on Solid-State Lighting in 2009. He received the Ta-You Wu Young Scholar Award from National Science Council Taiwan in 2007 and the Young Photonics Researcher Award from the OSA/SPIE Taipei chapter in 2007. He was elected as an OSA Fellow and an SPIE Fellow in 2012. 